\title{
Integrated Framework for the Design of Pipeline Systems Using Stochastic Optimisation and GIS tools
}

\author{
Eftychia C. Marcoulaki ${ }^{\mathrm{a}}$, Ioannis A. Papazoglou ${ }^{\mathrm{a}}$, Nathalie Pixopoulou ${ }^{\mathrm{b}}$ \\ ${ }^{a}$ System Reliability and Industrial Safety Laboratory (SRISL), National Centre for Scientific \\ Research “Demokritos”, PO Box 60228, Athens 15310, Greece \\ ${ }^{b}$ Penspen Limited, 405 Messogeion Avenue, Athens 15343, Greece \\ Corresponding author: \\ Dr. Eftychia C. Marcoulaki, E-mail: emarcoulaki@ipta.demokritos.gr \\ Telephone number: +30210 6503743, FAX number: +30210 6545496
}

\begin{abstract}
This work presents an optimisation framework for the routing and equipment design of main pipelines to be used for fluid transmission. There are many considerations in these design problems, involving various constraints, decisions and the associated costs for the construction, operation, maintenance, etc, of the system. In practice, engineers rely on experience, try out various design alternatives, and use simulators for engineering calculations, cost models, geographical information systems and equipment databases to identify promising options. The present approach proposes a systematic search for optimal and near-optimal solutions. The search is based on stochastic optimisation, and assumes that the same information and simulation tools as in the case of design by trial and error are available. An application example is used to demonstrate the approach and test the robustness of the optimal search using Simulated Annealing.
\end{abstract}

Keywords: Pipeline routing; GIS; Optimisation; Simulated annealing; Process design; Safety 


\section{Introduction}

During the last couple of years and despite the economic downturn, the number of oil and gas pipelines being planned and built is increasing, to meet the growing energy demands. Planned projects on December 2011 accounted for 119,938 miles of international pipelines, compared to 24,260 and 17,941 at the end of 2010 and 2009, respectively. Projects in various stages of construction had a 25\% increase compared to their 2010 and 2009 figures, accounted for 36,304 miles in 2011 (Tubb, 2011, 2010).

Many researchers have acknowledged the need for systematic methodologies to design optimal pipeline systems. Recent work on pipeline optimisation focused mainly on optimal layout development and minimisation of compressor fuel consumption. Wu et al. (2007) introduced a model to minimise the main pipeline length for compressor stations placed in series or forming a network. Ruan et al. (2009) presented a model that minimised the total pipeline and compressors investment cost. Middleton and Bielicki (2009) presented a model that estimated costs and solved for optimal spatial deployment for an efficient pipeline network. Rios-Mercado et al. (2006) formulated a minimisation problem for the fuel consumption of compressor stations in the natural gas pipeline transmission system. Baumrucker and Biegler (2010) considered the minimisation of operating fuel consumption in pipeline networks. Kabirian and Hemmati (2007) proposed a cost model combining installation and operating costs, to develop optimal network structures by selecting the type and location of pipeline and compressor stations. Grigoriev and Grigorieva (2009) also considered the location of control devices. Marseguerra et al. (2004) considered the optimal layout problem for a pipeline of given length in the presence of uncertainties in the failure rates of its constituent parts (i.e. pipes and joints). Wolfgang (2008) highlighted the need for combining investment and operating costs with system safety and maintainability.

The development of a pipeline system depends on the successful collaboration of different engineering disciplines to help decide on the best design alternative. Decisions taken at the early design stage will affect the investment and construction costs, the operational expenses and fuel consumption, and finally the maintenance, safety and environmental aspects of the system. The activities required at this stage include process modelling and simulation, geotechnical studies, pipeline route surveys, material studies and hydraulic modelling, always 
coupled with good engineering practice. Various geographical attributes of the area that the pipeline is expected to traverse appear as inputs to many of these activities. The proposed approach considers the above issues within a stochastic optimisation framework to conduct a systematic search for optimal design solutions. The search is supported by a set of generic procedures that allow the screening of alternative solutions. These rely on a set of perturbations for the successive generation of pipeline system designs. The implemented tool can be interfaced to available databases, geographical information systems and process simulators.

The paper is organised as follows. Section 2 describes the design problem. Section 3 discusses the design framework and the optimisation tool. Section 4 presents an application example and provides evidence on the robustness of the search. Section 5 concludes the work. The models used in the calculations of section 4 can be found at the appendix.

\section{Problem description}

Consider a fluid (compressible or incompressible) that needs to be transmitted from a production site to a consumption site. The data of the pipeline routing and design problem are:

- The transmitted fluid and its flow rate, and

- The location of the fluid supply and demand points.

Likewise to other engineering problems, optimisation objectives may include initial investment costs, energy consumption for the system operation, repair and maintenance costs, risks, environmental impact, as well as combinations of the above to form a multi-objective problem. The scope here is to obtain optimal solutions for the pipeline routing, the locations and design attributes of the pipeline network components, the system construction, operation and maintenance, according to one or a set of such objectives.

In practice, pipeline engineers try different configurations until a solution that can be considered satisfactory is obtained. Assessment of the solutions generated during this search requires a multitude of information to run cost and engineering models. The required information can roughly be divided into:

- geographical information on the terrain that the pipeline is traversing. This information is crucial in the design of main pipeline systems, considering that pipelines of the same length and capacity have completely different construction, operation and maintenance costs in different geographical locations. 
- data and coefficients required for engineering calculations. Similar information is required for other process design applications.

\section{Optimisation framework}

The optimisation framework developed here aims to simulate the design procedures followed in practice by pipeline engineers. Figure 1 illustrates the proposed optimisation algorithm. The algorithm starts with an initialisation step, before entering a loop where new solutions are generated, evaluated and compared to previously considered designs. The shaded boxes depend on the specific application. The dashed line encloses links to available information sources. The following subsections describe in more detail how we represent the system, how we evaluate the objectives for an instance of the system, how we generate new feasible instances of the system, and how we proceed on our search for optimal solutions. The discussion here is limited to single objective applications using standard Simulated Annealing (SA) optimisers. Note however that, the models and procedures described in sections 3.1-4 collaborate, in principle, with any single and multi-objective search that uses transitions between the system solutions (Marcoulaki and Papazoglou, 2010).

\subsection{Pipeline system representation}

This subsection discusses a general system representation, to support the screening of the solution space. The pipeline system is comprised of a single pipeline and various pressure control devices (PCD's). The PCD's include pumps or compressors, according to the properties of the transmitted fluid. A design instance is uniquely identified by knowing the exact routing of the pipeline, the exact locations of PCD's, and the exact design characteristics of all the equipment used in the system. Figure 2 is a simple illustration of a pipeline with three pumps, traversing a mountainous terrain containing a lake, a forest and a city. The red dashed line indicates a terrain boundary for the optimal search. Geological formations and types of land use may place specific restrictions and/or difficulties on the construction, operation and maintenance of the pipeline. The design of Figure 2 might not be optimal. It has segments inside the lake and over the mountain, where construction and maintenance are challenging. It is traversing through an area of special environmental concern, as well as a populated area where safety is very important and land is more expensive. This design is a feasible solution that the algorithm may visit during the optimal search. 
In the system representation assumed here, the pipeline is comprised of $N_{S}$ consecutive pipe segments and $N_{P}$ PCD's. The segments are allowed to have different length and orientation. They are represented by an ordered collection (list) of the coordinates, $\vec{v}_{i}$, of $N_{S}+1$ points on a three dimensional plane, where $\vec{v}_{i}=\left[x_{i}^{S}, y_{i}^{S}, z_{i}^{S}\right], i \in\left\{1,2, \ldots, N_{S}+1\right\}$. The $\vec{v}_{1}$ is the vector of coordinates at the beginning of the first segment. The $\vec{v}_{N_{S}+1}$ is the vector of coordinates at the end of the last segment $N_{S}$. This collection is denoted by $\boldsymbol{S}=\left\{\vec{v}_{i}\right\}$. For instance, the pipeline of Figure 2 has 17 segments represented by a list of 18 points. The locations of PCD's are given relatively to the pipeline by the set $\boldsymbol{P}=\left\{k_{j}\right\}, j=1,2, \ldots, N_{P}$, where $k_{j}$ denotes the pipeline segment connected to the device outlet. The pipeline of Figure 2 also has three pumps and $\boldsymbol{P}=\{1,5,14\}$.

Apart from their locations, each piece of equipment has specific design attributes. Attributes for each device $j$ are stored in the vectors $\overrightarrow{\boldsymbol{d}}_{j}^{P}$, and include the type, capacity or other features depending on the kind of PCD, e.g. the head if the PCD is a pump. For pipe segments, there are attributes concerning the entire segment (e.g. roughness, internal diameter), and others that vary along the pipe (e.g. pressure, slope). The former are stored in vector $\overrightarrow{\boldsymbol{d}}_{i}^{s}$, for each segment $i$. For the later, each segment $i$ is divided into congruent subsegments, and attributes for each subsegment $i_{S}$ are stored in vector $\overrightarrow{\boldsymbol{d}}_{i_{S}}^{S S}$. The subsegments have a user-specified maximum length.

To summarise the system representation assumptions, the pipeline is considered as single continuous line, approached by a series of consecutive linear segments. A single PCD can be placed on the starting point of each segment. For attributes that vary continuously along the pipe segments, each segment is sliced into subsegments of a user-defined maximum length. This is a reasonable assumption, since in practice the pipes come in straight pieces, whose length ranges between 12 and $18 \mathrm{~m}$ long depending on their manufacturer and deployment. The attribute profiles along the subsegments are linearised. This assumption is in accordance to the data accuracy, since the resolution in the GIS files may be at several meters.

\subsection{Simulation and evaluation of system objective(s)}

Using the representation described above, every instance of the pipeline system can be mapped to a set of $\left\{\boldsymbol{S}, \boldsymbol{P}, \overrightarrow{\boldsymbol{d}}_{j}^{P}, \overrightarrow{\boldsymbol{d}}_{i}^{S}, \overrightarrow{\boldsymbol{d}}_{i_{f}}^{S S}\right\}$ variables. Part of the information stored in this set relates to independent variables, and constitute the problem decision variables. The decision 
variables are derived through the optimal search, following the screening procedures described later. The rest are dependant variables, and their values can be estimated when the independent entities are known. For instance, the elevations, $z_{i}^{s}$, are derived from the pairs $\left[x_{i}^{S}, y_{i}^{S}\right]$, since geographical information is available. The required pipe thickness at any given point of the pipeline depends on the pipe internal diameter, the roughness and the transmitted fluid pressure. This section discusses the information and models required:

- to determine the dependant variables in the set, given the independent ones;

- to evaluate the system objective function value(s).

Adopting the classification of section 2 for the required information, engineering data and coefficients include the following:

- Physical property data of the transmitted fluid and the operating fuel. Note that, the method presented here can also be applied to multi-component fluids.

- Predictions on future prices for the procurement of operating fuel.

- Technical data obtained from equipment databases, including:

- design characteristics (e.g. available types, sizes, capacities, efficiencies, safety attributes) of pipes and PCD's;

- reliability and maintenance data (mean times to failure, mean times to repair, options for repair and maintenance schemes);

- unit costs for procurement, deployment, construction, installation, operation, maintenance, repair, inspection; etc.

Geographical information is obtained through links to GIS tools, to retrieve data on:

- elevation, slope, medium (soil/water), soil type etc;

- land use, population density, presence of other critical infrastructures (e.g. transportation network, industrial sites), and real estate costs;

- crossings with rivers, forests, natural heritage etc;

- factors affecting the construction, operation and maintenance costs of the pipeline network and its components;

- environmental condition and weather data;

- probability for natural disasters (e.g. earthquakes); etc.

The above information serves as input to process simulation models for hydraulic calculations, models to estimate energy consumptions, cost functions, models for system availabil- 
ity predictions etc. The collection of models depends on the specific design application, to meet the desired accuracy of modelling results and evaluate the user-defined set of system objective(s). The specific models used for the application example of section 4 can be found at the Appendices. Appendix A presents a model for pressure drop calculations commonly used by pipeline engineers, and Appendix B gives the cost models. These are general models with wide application range, however, other design problems may require different, more detailed or more specialised models. The optimiser used in this work and described in section 3.4 is not gradient-based, so there are no restrictions on the complexity the employed models or the types of their equations. Black-box models can also be accommodated.

Therefore, assumptions regarding the system simulation and evaluation of objectives depend on the specific application choices. Section 4.2 gives the assumptions for the design application example presented here.

\subsection{Set of perturbations}

The search for an optimal solution proceeds iteratively by generating a series of design instances. Each new design instance is the outcome of modifications applied on the decision variables of the current design in the series. The modifications are according to a set of perturbations. The choice of performing a specific perturbation is according to given probabilities distributions over a set of available alternative modifications. Perturbations should be simple, reversible and ensure that every feasible system design can be visited.

Perturbations can either be applied on (i) the characteristics of the current solution components, or (ii) the solution topological features. The perturbations of case (i) are applied to the decision variables of each piece of equipment. The following stepwise procedure is adopted in this case:

1. identify the decision variables and select randomly one of them. Selections are performed using user defined probability distribution functions (pdf's). For instance, select among the three pumps of Figure 2, with probability 1/3 each. Then select whether to modify the pump type or the pump capacity, with probability 0.8 for the capacity and 0.2 for the type. Let $w$ denote the selected variable.

2. identify the set of feasible values for variable $w$. Take for instance the pumps of Figure 2, and a given set of available pump types accompanied by their capacity ranges. According to the required pumping power, certain pump types or capacities may not 
be feasible for this particular pipeline.

3. select randomly a new value for variable $w$. If $w$ is the type of a pump, then a different type is selected among the feasible ones. If $w$ is the capacity of a pump, then a different capacity is selected among a range of feasible values. The selections are according to user specified pdf's.

More information on the procedure and the applied pdf's can be found in Marcoulaki and co-workers for integer (2000) and continuous $(1999,2001)$ variables.

Perturbations of case (ii) screen different options for the pipeline routing and the location of PCD's. Let a current pipeline with the characteristic sets $\boldsymbol{S}=\left\{\vec{v}_{i}\right\}, i \in\left\{1,2, \ldots, N_{S}+1\right\}$ and $\boldsymbol{P}=\left\{k_{j}\right\}, j \in\left\{1,2, \ldots, N_{P}\right\}$. A perturbation on the current pipeline returns a new design with $\boldsymbol{S}^{\prime}=\left\{\vec{v}_{i^{\prime}}^{\prime}\right\}, i^{\prime} \in\left\{1,2, \ldots, N_{S}^{\prime}+1\right\}$ and $\boldsymbol{P}^{\prime}=\left\{k_{j^{\prime}}^{\prime}\right\}, j^{\prime} \in\left\{1,2, \ldots, N_{P}^{\prime}\right\}$. The following perturbation cases are considered:

- Remove an existing point from the current pipeline. Figures 3.a-b illustrate this perturbation for a point not associated to a PCD (3.a) and when a PCD is involved (3.b). The first step is to select a point, $\vec{v}_{n}$, among the existing points $\left\{\vec{v}_{2}, \vec{v}_{3}, \ldots, \vec{v}_{N_{S}}\right\}$. The selection is performed randomly according to a uniform pdf. The fixed start and end points are excluded from the selection pool. The consecutive segments $\left\langle\vec{v}_{n-1}, v_{n}\right\rangle$ and $\left\langle\vec{v}_{n}, \vec{v}_{n+1}\right\rangle$ are replaced by the new segment $\left\langle\vec{v}_{n-1}^{\prime}, \vec{v}_{n}^{\prime}\right\rangle=\left\langle\vec{v}_{n-1}, \vec{v}_{n+1}\right\rangle$. Then $\boldsymbol{S}^{\prime}=\left\{\vec{v}_{1}, \vec{v}_{2}, \ldots, \vec{v}_{n-1}, \vec{v}_{n+1}, \ldots, \vec{v}_{N_{S+1}}\right\}$. If the deleted point, $\vec{v}_{n}$, is associated with a PCD $k_{m}=n$, the PCD is moved backwards, so that $k_{m}^{\prime}=n-1$.

- Relocate a point present in the current pipeline. Figures 3.c-d illustrate this perturbation for a point not associated to a PCD (3.c) and when a PCD is involved (3.d). A point, $\vec{v}_{n}$, is selected among the existing points $\left\{\vec{v}_{2}, \vec{v}_{3}, \ldots, \vec{v}_{N_{S}}\right\}$, according to a uniform pdf. The new location, $\vec{v}_{n}^{\prime}$, of the selected point is randomly selected within the locus $B \cap C$, where $B$ is the considered terrain, and $C$ is the disk with diameter $\left\langle\vec{v}_{n-1}, \vec{v}_{n+1}\right\rangle$. Note that, by enforcing that new point resides within the $\mathrm{C}$ area, we impose biases against the creation of loops. If $\vec{v}_{n}$ is associated with the PCD $k_{m}=n$, the device moves along with the point, so $k_{m}^{\prime}=n$, also.

- Add a new point to the current pipeline, as illustrated in Figure 3.e. A segment $\vec{v}_{n}-\vec{v}_{n+1}$ is selected randomly among the $N_{S}$ current segments $\left\langle\vec{v}_{1}, \vec{v}_{2}\right\rangle,\left\langle\vec{v}_{2}, \vec{v}_{3}\right\rangle, \ldots$, 
$\left\langle\vec{v}_{N_{S}}, \vec{v}_{N_{S}+1}\right\rangle$, according to a uniform pdf. A point, $\vec{v}_{n+1}^{\prime}$, is randomly selected within the locus $B \cap C$, where $B$ is the considered terrain, and $\mathrm{C}$ is the disk with diameter $\left\langle\vec{v}_{n}, \vec{v}_{n+1}\right\rangle$. Finally, the new point, $\vec{v}_{n+1}^{\prime}$, is introduced to the current solution, to yield a new pipeline with $\boldsymbol{S}^{\prime}=\left\{\vec{v}_{1}, \ldots, \vec{v}_{n}, \vec{v}_{n+1}^{\prime}, \vec{v}_{n+1}, \ldots \vec{v}_{N_{S}+1}\right\}$.

- Add new / remove existing PCD's in the current pipeline, as illustrated in Figure 3.f. A point, $\vec{v}_{n}$, is selected among the existing points $\left\{\vec{v}_{1}, \vec{v}_{2}, \ldots, \vec{v}_{N_{S}}\right\}$, according to a uniform pdf. Note that, the start point, $\vec{v}_{1}$, is now included in the selection pool. If the point already hosts a PCD, then the device is removed and the $\boldsymbol{P}$ set is properly modified. It is reminded that, type modifications are considered in the perturbations of case (i). If the point is not associated with PCD's, that a new device is added, and the $\boldsymbol{P}$ set is properly modified to include the selected location, $n$. Type and capacity for the new device are selected by applying perturbations of type (i) on the default values.

- Relocate PCD's in the current pipeline. This perturbation does not involve changes in the pipeline routing. It is performed as a combination of add/remove perturbations. First, an existing PCD, $m$, is removed, according to the perturbation for removing a PCD. Then, a new device $m^{\prime}$ with the characteristics of $m$ is introduced, according to the perturbation for adding a new PCD.

The perturbations require certain user-defined parameters. These include upper bounds on the number of pipe segments and PCD's, and probability distribution parameters. The bounds should be adequately large to allow flexibility in the search. Uniform probability distributions can be used to avoid creating biases.

\subsection{Feasibility of perturbed design}

The above perturbations may generate infeasible designs or designs containing loops in the pipeline. The loops can occur after perturbations on topological features. Solutions with loops are obviously inferior, therefore, not considered in the optimal search. Topological perturbations are followed by a loop detection procedure, and the perturbation is repeated if a loop is present in the perturbed design.

The following discussion considers infeasibilities in terms of the available PCD power. Consider for instance a pipeline with known routing and elevation profile. Let two pumps, one at the start and another at the middle of the pipeline. If the perturbations alter the length of the 
pipeline, the capacity of the PCD's needs to be adjusted, so that the pumping power input compensates for the pressure losses related to wall friction and changes in elevation downstream of each PCD. If the perturbations alter the location, number or type of the PCD's, then the capacities of the PCD's in the resulting design need to be properly adjusted. Likewise, when the PCD capacities are perturbed, the new design might have an excess or deficit of pumping power.

The adjustment actions follow a set of good practice rules, and they are performed in collaboration with the process simulation models discussed earlier. This collaboration is illustrated in Figure 1, where the simulator models are called before the new design is finalised. Let a user-defined lower bound on pressure, $p_{l b}$. The procedure is as follows:

1. calculate the pressure profile along the pipeline, and find the point of minimum pressure. The point of minimum pressure is always located at PCD inlets or at elevation maxima.

2. if the minimum pressure is found lower than $p_{l b}$, then the design is not feasible. We need to increase the pumping power before the minimum pressure point. First, we calculate the power required to increase the minimum pressure to $p_{l b}$. Then, this power is distributed among the pumps placed upstream of the minimum pressure point. The PDC capacities are increased accordingly.

3. an additional rule applied here is that the minimum pressure between successive PCD's is equal to $p_{l b}$. This rule aims to minimise the power losses. The procedure we use to reduce the pressures is the reverse of what described in step 2 .

4. Steps 1-3 are repeated until a feasible design is obtained.

If other infeasibilities need to be considered (e.g. upper bound on pressure), similar procedures can be developed.

\subsection{Optimiser}

The SA optimiser guides the optimal search along the solution space. Going back to Figure 1 , each perturbation is assessed stochastically according to the standard Metropolis criteria (Metropolis et al., 1953). These criteria compare the objective function values of the new and the current design solutions, and return a verdict on accepting or rejecting the perturbation. "Accept" means that the new design replaces the current design. "Reject" means that 
the current design is maintained. Then, another perturbation is applied, the perturbation is assessed etc. The verdict of the Metropolis criteria depends on the amount of improvement that $\mathrm{t}$ he perturbation brings to the objective, and on the value of a search control parameter, called the effective SA temperature. This control parameter is reduced throughout the search using a cooling schedule. The iterations performed at constant temperature constitute a homogeneous Markov chain, and the length of this chain is user defined. Marcoulaki and Kokossis $(1999,2000)$ provide details on the SA cooling schedule, and the rules for search termination and propagation used here.

The standard SA is a well established heuristic method for global optimization. It has a very strong mathematical background on Markov processes, and takes advantage of their convergence properties. Consequently, SA is expected to converge to a statistical distribution of final values for the objective function. Aarts and van Laarhoven (1985) and Gidas (1985) provided proofs for asymptotic convergence to the vicinity of the global optimum. The final distribution can be sampled by running several computational experiments with different seeds for the random number generator. Marcoulaki and Kokossis (1999) showed that by decreasing the annealing speed and increasing the chain length the average of the final distribution moved closer to the globally optimal objective function value, and the deviation tended to zero. Their convergence results were also independent of the initial state guess. Section 4 presents a similar analysis for pipeline systems.

Note that, though the values of the final solution objectives may be very close, the underlying design choices could be very different. So the final result is not a single solution, but a set of solutions which are very similar in terms of their e.g. cost, but differ in their structural and operational features. This is very important in practical applications, like process systems engineering problems. Engineers can benefit from having a set of promising designs, to apply non-quantifiable criteria in choosing the design to be finally implemented.

\section{Design application example}

\subsection{Problem description and design cases}

The proposed methodology is illustrated using the design of a main pipeline system for the transmission of $160 \mathrm{kBPD}$ crude oil of known properties between two given transmission points. The pipeline needs to satisfy the following set of objectives: 
- initial investment costs for pipeline network components,

- construction costs, including deployment and installation activities,

- operating costs based on the pump operating fluid requirements, and

- costs for scheduled maintenance, inspection and repairs.

The above objectives are aggregated in a single cost function using a life time of 20 years. The considered region for the pipeline route has the form of a 35 by $40 \mathrm{~km}$ orthogonal rectangle. Figure 4a gives the ground elevation contours of this region. Ground elevations at the start and destination points are $292.3 \mathrm{~m}$ and $70.2 \mathrm{~m}$, respectively. The length of a pipeline with straight $\mathrm{x}-\mathrm{y}$ projection between the start and destination points is $40,317 \mathrm{~m}$. This minimum length pipeline traverses over a mountainous area, with a peak of $482 \mathrm{~m}$. Specific geographical information is available, and includes ground elevation, soil type, population density, real estate data, presence of other infrastructures. The considered region contains a populated area and major transportation infrastructures. Figure $4 \mathrm{~b}$ illustrates the population density contours and the motorways.

Two design cases, A and B, and two sets of computational experiments are considered. Case A considers only the geological information (slopes, elevations, soil type etc; see Figure 4a). Case B also considers additional information available on the region, like population, land use, and other infrastructures (see Figure 4b). The first set of computational experiments is to test the performance and convergence characteristics of the design tool. The second set is to investigate how the final designs are affected by the geographical information considered.

The models for equipment costing can be found at the appendix. The model parameters are reported on Tables 1 and 2. Table 1 presents cost coefficients and base costs. Certain values are based on past data from Penspen and SRISL projects. In Table 2, the $F_{i, K}, K \in\{I, L, C\}$ are factors for equipment sizing and costing. They depend on the geographical characteristics of the location where the equipment is to be placed.

The pipe material is commercial steel. Price and properties are found in Table 1. A range of suitable pipe diameters is determined according to the allowable pressure drops and transmitted fluid velocities. Standard engineering practice sets the allowable ranges for velocity and pressure drop at $1.5-2.5 \mathrm{~m} / \mathrm{s}$ and $0.01-0.05 \mathrm{bar} / 100 \mathrm{~m}$, respectively. The range of internal diameters obeying these constraints is between 17.9" and 19.8". The internal diameter 
within the pipeline is herein fixed to 19.25 ". This corresponds to the internal diameter of a 20 " nominal pipe with standard pipe thickness 0.75 ". The resulting oil flow resides in the turbulent region. The fluid pressure drop is $0.03528 \mathrm{bar} / 100 \mathrm{~m}$, and its velocity equals 1.568 $\mathrm{m} / \mathrm{s}$ (see Appendix A). The pipe wall thickness is adjusted according to the crude oil pressures developed along the pipe, and the class location factors (see Appendix B, Table 2).

Pump locations are not considered in the present application, since the pipelines are quite short and the pressure drop is low, giving a maximum head less than $300 \mathrm{~m}$. This means that, a single pump at the source point is sufficient to provide the required power. The pump head is adjusted to meet the static and frictional pressure drops along the route of each pipeline design visited during the optimal search, as discussed in section 3.4. The operating fuel is natural gas. The pump calculations are according to the models in the Appendices and the data in Table 1.

\subsection{Convergence results and discussion}

The first set of computational experiments is to test the effect of search control parameters on the convergence of the annealing optimiser.

In brief, the parameters controlling the optimiser are the following:

- the initial SA temperature. This is automatically set at ten times the objective function value of a randomly generated design.

- the cooling control parameter. This is always set at 0.05 .

- the length of the homogeneous Markov chain (i.e. number of iterations at each constant SA temperature interval).

Computational experiments are run for increasing Markov chain lengths. According to theory (Aarts and van Laarhoven, 1985; Gidas, 1985), when the chain length increases, the search converges to the globally optimal objective function value with higher probability. In practice, this means that the probability distribution of the objective function values of the final solutions is narrower and moves closer to the global optimum. Note that, the number of possible solutions in the present application is well above $10^{185}$, so the convergence to the global optimum can not be confirmed by exhaustive enumeration.

The results are obtained for design case A, with Markov chain lengths 6, 10, 20 and 30. The stochastic optimiser is run several times to obtain a sufficient sample of final results. The 
initialisation is performed randomly and there is no recollection of the initial state. To be more specific, at the beginning of each run the algorithm starts with a straight pipe between the given supply/demand points, and applies a series of 20 random perturbations, to generate an initial guess for the run. The run sample size is 16 . Since the sample size is small and the distribution is not necessarily normal, the following statistical analysis is based on the sample median and quartiles. The quartile variation coefficient, $Q V$, is also used as a measure of dispersion:

$$
Q V=\frac{Q_{3}-Q_{1}}{Q_{3}+Q_{1}}
$$

where $Q_{1}$ and $Q_{3}$ are the lower and upper quartiles, respectively (Evans et al., 2000).

Figure 5a shows that the number of simulations per run increases exponentially as the chain length increases. A five-times increase in the chain length (from 6 to 30) yields a fortytimes increase in the computational time. The quartile variation coefficient fluctuates around $9 \%$, without an evident trend between the dispersions in computational times and the chain length.

Figure $5 \mathrm{~b}$ shows that the median of the length of the pipeline decreases by $3.6 \%$ as the chain goes from 6 to 30. At the same time, the quartile variation coefficient starts at $4.6 \%$ when the chain length is 6 , and drops below $1 \%$ and $0.5 \%$ at chain lengths 20 and 30, respectively. Note that, the results feature pipeline lengths well above the minimum distance between inlet and outlet point. This is observed even at the results for chain length 6 . Figure 6 illustrates the final routes obtained using different chain lengths. Each route is represented by a line connecting the start and end points. Note that, the pipe features at each point along these lines depend on the geographical information and the transmitted fluid conditions.

Figure $5 \mathrm{c}$ shows the median and the quartile variation coefficient of the objective function values. The figure also shows the cost of a design optimised for distance rather than cost. This design features the minimum pipeline length, and costs $\$ 45,352 \mathrm{~K}$. The total costs at Markov chain 6 are $1 \%$ to $5 \%$ above the cost of the minimum pipeline length solution. As the chain increases to 10 , the costs drop below that of the minimum pipeline length solution. In absolute numbers, the differences between the cost medians at chains 6 up to 30 from the cost of the minimum length design drop from $+\$ 750 \mathrm{~K}$ down to $-\$ 500 \mathrm{~K}$. As the chain increases by 4 times (from 6 to 30), the dispersion in the results drops by almost 20 times, 
from $1.96 \%$ to $0.13 \%$. It is important to highlight that, as the chain length increases, the results converge to the vicinity of the best objectives visited during the analysis.

The obtained results are supported by the theoretical background of the Markov processes involved in SA and confirm the expected trends for convergence (Aarts and van Laarhoven, 1985; Gidas, 1985). As the chain increases, the objective function values gather closer around the best values visited during the search. This trend is independent of any initial guess, since the above results are obtained starting with randomly chosen initial designs. The convergence results agree with previous work on the robustness of the annealing algorithm (Marcoulaki and Kokossis, 1999). These trends are observed with perturbations that are only applicable to pipeline systems and have not been tested elsewhere. In the present case study, the pipeline length also converges to a target value. This is a coincidence, and indicates that the minimum cost is achieved by designs featuring similar total pipe length.

It is important to highlight that, the performance of the optimisation tool is not hindered by non-linearities in the model equations or discontinuities in the design variables. The tool can, thus, accommodate more detailed cost models and additional design criteria, if relevant data are available.

\subsection{Final pipeline designs and discussion}

Results are obtained for design cases A and B. The Markov chain length is 20. The analysis in subsection 4.2 indicates that this chain length provides adequate convergence. Other search control parameters and the initialisation process are according to subsection 4.2. Table 3 itemises the statistics for final costs, and also reports the statistics for the total pipeline lengths and the number of simulations performed during the optimal search. Values are presented with accuracy of at least three significant digits. Operating and maintenance (including repair) costs are considered over the 20 years lifetime of the project.

According to Table 3, the results for case B have increased equipment investment costs. This is due to additional safety considerations, and the special construction activities needed when the pipeline segments pass though populated areas (ASME, 2010). Pipeline construction and maintenance costs are increased by about 7\% compared to case A. Investment and real estate costs are very low in both cases, but the increase from case A to case B is over $40 \%$. Pump costs are affected, since the pressure profiles obtained for the two cases are different. Pump investment and maintenance costs are increased by $11 \%$, while the increase in 
pump fuel costs is over $13 \%$. The dispersion in the obtained pipeline costs increases by $42-$ 44\%. The dispersion in the pump costs decreases by $39-40 \%$, since the B-case results have similar pumping pressure requirements. Despite the range of $Q V$ at specific costs, the dispersion in the total costs is similarly low for both design cases. This provides additional proof on the good convergence properties of the algorithm.

For case A, the optimisation tool delivers two sets of final solutions (see Figure 6c). In the first set, the designs are close to the route of minimal length and go over the mountainous area. The designs in the second set suggest a quite longer route at lower elevations. The designs in the two sets are similar in terms of total costs, since, the construction penalties and the increased pumping costs in the first set are counterbalanced by the costs of designing a longer pipeline in the designs of the second set. The set of optimal designs generated for case B indicates a strong preference to a route around the mountains, to avoid the populated area (see figure 7).

Clearly, the proposed tool succeeds in producing near-optimal designs that satisfy the requirements of each case. In case A, safety is not an issue and construction difficulties include only the slope, thus the dominant cost factors are the pipeline length and the elevation differences. The two sets of final designs reflect the trade-offs between these two factors: shorter pipelines and higher pressure head versus longer pipelines going around the mountain. This explains the variations in the pump investment, operation and maintenance costs (see Table 3). In case B, the optimal decision is just to avoid the populated area, by designing a longer pipeline running very close to the city borders. Note that, computational experiments with relaxed design factors give final designs between the two extremes of cases $\mathrm{A}$ and $\mathrm{B}$. In these cases, pipelines are shorter and a part of them passes through the city. This part becomes larger as the population density is decreased.

Apart from the cost figures and the routing, the final solutions provide detailed information on the design characteristics of every pump and pipe segment used in the system. Figure 8 shows the pipe wall thickness profiles for three of the final designs. Designs $D_{1}$ and $D_{2}$ are for case $A . D_{1}$ traverses over the mountain, the pipeline length is $40,344 \mathrm{~m}$, the total pressure drop is 14.2 bar and the required pump head is 27.0 bar. $\mathrm{D}_{2}$ goes around the mountain using a slightly longer pipeline, with length $41,495 \mathrm{~m}$ and total pressure drop $14.6 \mathrm{bar}$. The required pump head drops to 22.6bar, since the pipeline is at lower elevations. This design exhibits increased wall thicknesses at the start and end points, where the pipe internal pressure is 
higher. The lowest pipe wall thickness is observed at the highest elevation along the pipeline. Design $\mathrm{D}_{3}$ is for case $\mathrm{B}$. The pipe length here is $44,236 \mathrm{~m}$, the pressure drop is $15.6 \mathrm{bar}$ and the required pump head is 26.1 bar. Though the pressures are lower than $D_{2}$, the presence of habituated regions and critical infrastructures calls for special design and safety considerations, and the wall thickness increases.

\section{Conclusions}

This manuscript presents a new approach for the design of main pipeline systems, used for the transmission of fluids, like oil, natural gas, water or biofuel. In practice, pipeline engineers start from the geographical attributes of the area that the pipeline is expected to traverse, they develop routes by trial and error, and use various rules and models for engineering calculation and the selection of equipment. The proposed approach aims to propose a systematic search for optimal solutions using the same information and simulation tools. To achieve that, advanced optimisation technologies are integrated with geographical information, process simulators and equipment databases. The implemented optimisation tool aims at real life applications, and takes into account hydraulics, equipment cost, reliability, operation and maintenance features, information on the landscape and land use etc.

The approach is demonstrated through a successful application on the design of a pipeline traversing through a mountainous terrain. Different sets of computational experiments test the performance and convergence characteristics of the design tool, and investigate how the final designs are affected by the geographical information considered. Supported by the theory behind Simulated Annealing processes, the obtained results provide evidence that the proposed framework converges to a set of final solutions in the vicinity of the global optimum. As expected, the use of different geographical data affects the investment, construction, operation, inspection and maintenance costs, and calls for different optimal pipeline routing and equipment design features. Current work considers extensions of the tool to take into account pipe corrosion phenomena.

\section{Appendix A: Pressure calculations}

This section describes the models used to calculate the pressures observed along a given pipeline design generated during the optimal search procedure. The variables and parameters in the following equations are in SI units (unless otherwise stated). Consider two points A 
and $\mathrm{B}$ in a known pipeline, with pressures $p_{A}$ and $p_{B}$, respectively. The pressure difference between $\mathrm{B}$ and $\mathrm{A}$ is formulated as:

$$
\Delta p_{A B}=p_{B}-p_{A}=p_{A B, C}-\Delta z_{A B} \cdot \rho_{A B} \cdot g-L_{A B}^{e q} \cdot \delta p_{A B}
$$

where $p_{A B, C}$ is the pressure head provided by PCD's placed along the AB segment. The second term is the static pressure loss due to elevation increase from point $\mathrm{A}$ to $\mathrm{B}$, where $\rho_{A B}$ is the mean fluid density, $g$ is the gravitational force and $\Delta z_{A B}$ is the elevation difference between points $\mathrm{B}$ and $\mathrm{A}$.

The last term denotes the pressure drop due to frictional forces, where $L_{A B}^{e q}$ is the total equivalent length of the segment $\mathrm{AB}$ and $\delta p_{A B}$ is the unit pressure drop due to friction. The total equivalent length is calculated as the actual segment length plus the length equivalents for gates and elbows present along $\mathrm{AB}$. The segment $\mathrm{AB}$ is a straight pipe starting from $\left[x_{\mathrm{A}}\right.$, $\left.y_{\mathrm{A}}, z_{\mathrm{A}}\right]$ and finishing at $\left[\begin{array}{lllll}x_{\mathrm{B}}, & y_{\mathrm{B}}, & z_{\mathrm{B}}\end{array}\right]$, so the actual length is $L_{A B}=\sqrt{\left(x_{B}-x_{A}\right)^{2}+\left(y_{B}-y_{A}\right)^{2}+\left(z_{B}-z_{A}\right)^{2}}$. For incompressible fluids, like oil, the unit pressure drop is calculated from Darcy's correlation (Crane, 2009):

$$
\delta p_{A B}=\frac{\rho_{A B} \cdot f_{A B} \cdot u_{A B}{ }^{2}}{2 \cdot d_{A B}}
$$

where $d_{A B}$ is the pipeline internal diameter, $f_{A B}$ is the Fanning friction factor, $u_{A B}$ is the fluid velocity $u_{A B}=4 \cdot Q_{A B} /\left(\pi \cdot d_{A B}{ }^{2}\right)$, and $Q_{A B}$ is the volumetric flow rate of the transmitted fluid in segment AB. Darcy's formula is valid for:

- laminar flow, with $R e<2000$ (Reynolds' number), where $f_{A B}=64 / R e$

- turbulent flow, with $R e>4000$, where $f_{A B}$ depends on $R e$ and the roughness of the pipe walls, $r$, and $f_{A B}$ is taken from Moody's diagram (Crane, 2009).

\section{Appendix B: Cost model equations}

This section describes the cost models used in the design application example. The variables and parameters in the following equations are in SI units (unless otherwise stated) and the costs are in USD. 


\section{B.1. Pipeline cost model}

According to section 3.2, the pipeline is hereby represented as a series of successively interconnected straight segments on the $x-y$ plane. Each segment point has its own geographical attributes (e.g. elevation, soil type, land use), equipment characteristics (e.g. diameters, roughness) and conditions (e.g. pressure). For cost evaluations, segments are further divided into subsegments using a user defined maximum length, $l_{\max }$.

Let a pipeline of $N_{S}$ subsegments. The total pipeline cost $C S T$ is formulated as the sum:

$$
C S T=\sum_{i=1}^{N_{S}} C S_{i, I}+C S_{i, L}+C S_{i, C}+C S_{i, M}+C S_{i, R}
$$

where the $C S_{i, I}, C S_{i, L}, C S_{i, C}, C S_{i, M}$, and $C S_{i, R}$ denote the costs of investment, land, construction, maintenance and repair, respectively, for subsegment $i \in\left\{1,2, \ldots, N_{S}\right\}$.

The cost of investment is largely dominated by the cost of steel, and is calculated as follows:

$$
C S_{i, I}=\mathrm{c}_{\mathrm{S}} \cdot m_{S, i} \cdot L_{i}
$$

where $c_{S}$ is the unit cost of steel, $m_{S, i}$ is the required steel mass per meter of $i$, and $L_{i}$ is the actual length of segment $i$. The value of $m_{S, i}$ is given by:

$$
m_{S, i}=\pi \cdot t_{i} \cdot\left(d_{i}+t_{i}\right) \cdot \rho_{S}
$$

where $\rho_{S}$ is the density of steel, $d_{i}$ is the pipe internal diameter, and $t_{i}$ is the pipe wall thickness. The wall thickness should comply with safety regulations and/or other design requirements, and is evaluated according to ASME (2010):

$$
t_{i}=\frac{p_{i}^{*} \cdot d_{i}}{2 \cdot s \cdot F_{i, I}}
$$

where $p_{i}^{*}=F_{p} \cdot p_{i}^{\text {max }}$ is the design pressure based on the maximum pressure $p_{i}^{\text {max }}$ observed in $i$ and a safety factor for pressure, $F_{p}$; and $s$ is the specified minimum yield strength of the pipe. The $F_{i, I}$ is a basic design factor depending on the location class of the area along subsegment $i$.

Location classes are defined according to the "number and proximity of buildings intended for human occupancy and other characteristics that are considered when prescribing design 
factors for construction, operating pressures, and methods of testing pipelines and mains located in the area and applying certain operating and maintenance requirements" (ASME, 2010). In brief, the location classes are:

- class location 1, division 1: any 1-mile section with less/equal to 10 buildings

- class location 1, division 2: 1.1 with extra safety or special construction difficulty

- class location 2: any 1-mile section with number of buildings between 10 and 46

- class location 3: any 1-mile section with more/equal to 46 buildings

- class location 4: any 1-mile section with multi-storey buildings, dense/heavy traffic

In the absence of specific real estate information, land costs where the pipes will be installed are estimated as:

$$
C S_{i, L}=F_{i, L} \cdot C S_{B, L} \cdot \delta_{i}\left(p_{i}^{*}\right) \cdot L_{i}
$$

where $c S_{B, L}$ is a base (minimum) land $\operatorname{cost} ; \delta_{i}$ is the hazard distance, associated to the transmitted fluid and the pipe pressure; and $F_{i, L}$ is a land cost adjustment factor.

Construction and installation costs are formulated as follows:

$$
C S_{i, C}=c S_{B, C} \cdot\left(d_{i}+2 \cdot t_{i}\right) \cdot F_{i, C} \cdot L_{i}
$$

where $c s_{B, C}$ is the base construction/installation cost; and $F_{i, C}$ is the corresponding design factor, to account for special restrictions and difficulties that call upon additional expenses.

Maintenance strategies aim to improve system reliability, avoid emergency shutdowns and equipment down time, thus increase the system availability. The maintenance and inspection costs over $T$ years of operation for subsegment $i$ is formulated as:

$$
C S_{i, M}=T \cdot C S_{i, M} \cdot L_{i}
$$

where $c s_{i, M}$ is the cost of the annual maintenance per meter of $i$, which is usually considered proportional to the initial costs of investment and construction, thus:

$$
C s_{i, M}=b_{i, M} \cdot C M_{i, M}
$$

where $b_{i, M}$ is a coefficient derived from available pipe maintenance data. In practice, these data can be provided by equipment manufacturers or be based on past experience. 
Repairs on pipes are usually performed by replacing the faulty segment, thus the total repair costs for subsegment $i$ over the $T$ years of pipeline operation are given as:

$$
C S_{i, R}=T \cdot N S_{i, f} \cdot\left(C S_{i, I}+C S_{i, C}\right)
$$

where $N S_{i, f}$ is the number of failures expected to occur annually per meter of $i$. Reliability data are given by the manufacturer, and industrial sources of reliability figures derived from equipment datasheets.

\section{B.2. Pressure control device cost model}

Pumps and compressor stations provide the necessary pressure for the fluid to be transmitted through the pipes. The application example presented in section 4 does not involve compressors, so only the pump model is presented here.

For a system of $N_{P}$ pumps, the total pump cost $C P T$ is the sum of the costs for each pump $j, j$ $=1,2, \ldots, N_{P}$ :

$$
C P T=\sum_{j=1}^{N_{P}} C P_{j, I}+C P_{j, F}+C P_{j, M}+C P_{j, R}
$$

where the $C P_{j, I}, C P_{j, F}, C P_{j, M}$ and $C P_{j, R}$ denote the costs of investment, operation, maintenance and repair, respectively, for pump $j$.

The pump investment cost includes procurement and construction expenses, and is derived as the sum of the main pump cost, $C P_{j, P}$, and the driver cost, $C P_{j, D}$ :

$$
C P_{j, I}=C P_{j, P}+C P_{j, D}
$$

Since data for large pumps are not openly available, investment costs can be based on vendor offers. A simple power formula can be used to calculated the costs of pump $j$, considering a base pump of known head $H_{B}$, main cost $C P_{B, P}$ and driver cost $C P_{B, D}$ :

$$
\frac{C P_{j, P}}{C P_{B, P}}=\left(\frac{H_{j}}{H_{B}}\right)^{a_{P}} \text { and } \frac{C P_{j, D}}{C P_{B, D}}=\left(\frac{H_{j}}{H_{B}}\right)^{a_{D}}
$$

where $a_{P}, a_{D}$ are fixed coefficients, and $H_{j}$ is the head of pump $j$, formulated as:

$$
H_{j}=\frac{p_{j}}{g \cdot \rho_{j}}
$$


where $p_{j}$ is the output pressure of pump $j ; g$ is the gravitational force; and $\rho_{j}$ is the mean density of the transmitted fluid in pump $j$.

The pump operating costs are dominated by the cost of pumping fuel, therefore:

$$
C P_{j, F}=V_{j, F} \cdot c_{F}
$$

where $c_{F}$ is the unit fuel cost; and $V_{j, F}$ is the total fuel volume consumed in pump $j$ during the period of the pipeline operation, calculated as:

$$
V_{j, F}=\frac{Q_{j, F} \cdot T \cdot \tau}{\eta_{j}}
$$

where $\eta_{j}$ is the efficiency of pump $j ; T$ denotes the operation time; $\tau$ is a coefficient for unit transformation; and $Q_{j, F}$ is the fuel volumetric flow rate given by:

$$
Q_{j, F}=\frac{P_{j}}{H V_{F}}
$$

where $H V_{F}$ is the heating value of the fuel; and $P_{j}$ is the required pumping power given as:

$$
P_{j}=Q_{j} \cdot p_{j}
$$

where $Q_{j}$ is the volumetric flow rate of the transmitted fluid in pump $j$.

Given a cost $c p_{j, M}$ for annual pump maintenance, the pump maintenance cost is evaluated as:

$$
C P_{j, M}=T \cdot c p_{j, M}
$$

Let $c p_{j, M}$ be the expected mean cost of each repair, then the total cost due to equipment failure is formulated as:

$$
C P_{j, R}=T \cdot N P_{j, f} \cdot c p_{j, R}
$$

where $N P_{j, f}$ is the expected number of pump equipment failures, given as $N P_{j, f}=T P_{j, f}{ }^{-1}$, with $T P_{j, f}$ being the mean time between failures for pump $j$ provided by the pump manufacturer.

The costs $c p_{j, M}$ and $c p_{j, R}$ are usually considered proportional to $C P_{j, I}$, thus: 


$$
c p_{j, M}=a_{j, M} \cdot C P_{j, I} \text { and } c p_{j, R}=a_{j, R} \cdot C P_{j, I}
$$

where $a_{j, M}$ and $a_{j, R}$ are coefficients based on available data on pumps similar to $j$.

\section{References}

Aarts EHL, van Laarhoven PGM. Statistical Cooling: A General Approach to Combinatorial Optimization Problems. Philips Journal of Research 1985; 40: 193-226

Annual Energy Review 2009, Report No. DOE/EIA-0384 (2009), Released August 2010 [available at http://www.eia.doe.gov/aer ]

American Society Mechanical Engineers (ASME). B31.8 - 2010 Gas Transmission and Distribution Piping Systems, 2010

Baumrucker BT, Biegler LT. MPEC strategies for cost optimization of pipeline operations. Computers Chemical Engineering 2010; 34: 900-913

Crane Co., Flow of Fluids Through Valves, Fittings and Pipe. Technical Paper No. 410M, Crane Co., Stamford, 2009

Evans M, Hastings N, Peacock B. Statistical Distributions, $3^{\text {rd }}$ ed. New York, Wiley, 2000

Gidas B. Nonstationary Markov chains and convergence of the annealing algorithm. Journal of Statistical Physics 1985; 39 (1/2): 73-131

Grigoriev A, Grigorieva NV. The valve location problem: Minimizing environmental damage of an oil spill in long oil pipelines. Computers and Industrial Engineering 2009; 57: 976-982

Jo Y-D, Crowl DA. Individual risk analysis of high pressure natural gas pipelines. Journal of Loss Prevention in the Process Industries 2008; 21: 589-595

Kabirian A, Hemmati MR. A strategic planning model for natural gas transmission networks. Energy Policy 2007; 35: 5656-5670

Marcoulaki E, Linke P, Kokossis A. Design of separation trains and reaction-separation networks using stochastic optimisation methods. Chemical Engineering Research and Design 2001; 79(1): 25-32.

Marcoulaki EC, Kokossis AC. Scoping and screening complex reaction networks using stochastic optimization. AICHE J. 1999; 45(9): 1977-91 
Marcoulaki EC, Kokossis AC. On the development of novel chemicals using a systematic synthesis approach. Part I - Optimisation framework. Chemical Engineering Science 2000, 55 (13): 2529-2546

Marcoulaki EC, Papazoglou IA. A dynamic screening algorithm for multiple objective simulated annealing optimization; Computer-Aided Chemical Engineering 2010; 28: 349-354.

Marseguerra M, Zio E, Podofillini L. Optimal Reliability/Availability of Uncertain Systems via Multi-Objective Genetic Algorithms. IEEE Transactions on Reliability 2004; 53 (3), $424-434$

Metropolis N, Rosenbluth AW, Rosenbluth MN, Teller AH, Teller E. Equation of State Calculations by Fast Computing Machines. Journal of Chemical Physics 1953; 21 (6): 10871092

Middleton RS, Bielicki JM. A scalable infrastructure model for carbon capture and storage: SimCCS, Energy Policy 2009; 37 (3): 1052-1060

Rios-Mercado R, Seongbae K, Boyd A. Efficient operation of natural gas transmission systems: A network-based heuristic for cyclic structures. Computers \& Operations Research 2006; 33: 2323-2351

Ruan Y, Liu Q, Zhou W, Batty B, Gao W, Ren J, Watanabe T. A procedure to design the mainline system in natural gas networks. Applied Mathematical Modelling 2009; 33: 30403051

Tubb R. Pipeline \& Gas Journal's 2010 International Pipeline Construction Report. Pipeline \& Gas Journal 2010; 237 (2) [available from http://pipelineandgasjournal.com]

Tubb R. Pipeline \& Gas Journal's 2011 Worldwide Pipeline Construction Report. Pipeline \& Gas Journal 2011; 238 (1) [available from http://pipelineandgasjournal.com]

Wolfgang K. Critical infrastructures at risk: A need for a new conceptual approach and extended analytical tools, Reliability Engineering \& System Safety 2008; 93: 1781-1787

Wu Y, Lai KK, Liu Y. Deterministic global optimization approach to steady-state distribution gas pipeline networks. Optimization \& Engineering 2007; 8: 259-275 


\section{List of acronyms}

ASME _ American Society of Mechanical Engineers

GIS _ Geographical Information System

PCD _ Pressure Control Device

SA __ Simulated Annealing

SRISL _ System Reliability and Industrial Safety Laboratory

USD _ United States Dollar

\section{Nomenclature}

$a \_$cost coefficient for PCD

$b_{\text {_ }}$ cost coefficient for pipe subsegment

$c \_$unit cost

cS _ _ annual and/or base cost for subsegments

$C P$ _ cost element for PCD

$C P T$ _ total pump cost

$C S \_$cost element for subsegment

$C S T \_$total pipeline cost

$d_{\text {_ }}$ pipe internal diameter

$\overrightarrow{\boldsymbol{d}}$ _ vector of attributes

$f \ldots$ Fanning friction factor

$F$ _ design factor for equipment sizing and costing

$g$ _ gravitational force

$H_{\text {_ }}$ pump head

$H V$ _ heating value

$k_{j}$ _ pipeline segment connected to the outlet of $\operatorname{PCD} j, k_{j} \in\left\{1,2, \ldots, N_{S}\right\}$

$l_{\text {max _ maximum subsegment length (provided by the user) }}$ 
$L \_$_ actual length of segment/subsegment

$L_{A B}^{*} \_$total equivalent length of the segment $\mathrm{AB}$

$m$ _mass

$N P_{f}$ _ expected rate of failures for pumps

$N S_{f}$ _ expected rate of failures for segments

$\boldsymbol{P}=\left\{k_{j}\right\}, j=1,2, \ldots, N_{P}$ _ set of locations of PCD's relatively to the pipeline

$p$ _ pressure

$p^{*}$ _ pipe design pressure

$p_{A B, C}$ _ pressure head provided by PCD's placed along $A B$

$p_{l b} \ldots$ lower bound on pressure along the pipeline

$Q$ _ volumetric flow rate of the transmitted fluid

$Q_{1}$ _ lower quartile

$Q_{3}$ _ upper quartile

$Q V$ _ quartile variation coefficient, $Q V=\frac{Q_{3}-Q_{1}}{Q_{3}+Q_{1}}$

$r_{\text {_ }}$ pipe roughness

Re _ Reynolds' number

$s_{\text {_ }}$ pipe minimum yield strength

$\boldsymbol{S}=\left\{\vec{v}_{i}\right\}, i \in\left\{1,2, \ldots, N_{S}+1\right\}$ _ ordered list of pipeline segment coordinates, $\vec{v}_{i}$

$t_{\text {__ }}$ pipe wall thickness

$T_{\text {_ design operation lifetime }}$

$T P_{f} \_$mean time between failures for pumps

$u \_$transmitted fluid velocity

$V$ _ volume

$w^{\prime}$ _ instance of a variable or set $w$ after a random perturbation is applied

$w^{*}$ _ actual value of variable $w$ 
$z$ _ point elevation

$\delta$ _ hazard distance

$\delta p_{A B}$ _ unit pressure drop due to friction along $\mathrm{AB}$

$\Delta p_{A B}$ _ pressure difference on segment $A B$

$\Delta z_{A B}$ _ elevation difference between points B and A

$\eta \ldots$ pump energy efficiency

$\vec{v}_{i}=\left[x_{i}^{S}, y_{i}^{S}, z_{i}^{S}\right], i \in\left\{1,2, \ldots, N_{S}+1\right\}$ _ vector of coordinates in $\mathrm{x}-\mathrm{y}-\mathrm{z}$ axes

$\rho$ _ density

$\tau$ __ unit conversion

\section{Subscripts and superscripts}

$A B$ _ a pipeline segment, between points $A$ and $B$

$B$ _ base cost

C_ construction

$D_{\text {_ }}$ pump driver

$f \ldots$ failures

$F \_$PCD fuel

$I \_$investment

$i \_$index for pipe segment, $i \in\left\{1,2, \ldots, N_{S}\right\}$

$i_{S} \ldots$ index for subsegment of segment $i, i_{S} \in\left\{1,2, \ldots, N_{i}\right\}, N_{i}=\operatorname{int}\left[\frac{\left|\vec{v}_{i+1}-\vec{v}_{i}\right|}{l_{\max }}\right]+1$, where int $[w]$ denotes the integer part of $w \in \mathbb{R}$

$j \_$index for PCD, $j \in\left\{1,2, \ldots, N_{P}\right\}$

$L \_$land / location

$m \_$index for selected PCD, $m \in\left\{1,2, \ldots, N_{P}\right\}$

$M_{\text {_ }}$ maintenance

$\max \_$maximum 
$n \ldots$ index for selected pipeline segment or point, $n \in\left\{1,2, \ldots, N_{S}\right\}$

$N_{P}$ _ number of PCD's in the pipeline

$N_{S}$ _ number of consecutive pipe segments in the pipeline

$P_{-}$PCD, pump

$R_{\text {_ repair }}$

$s_{\text {_ }}$ pipe material (steel)

$S_{\text {_ }}$ pipeline segment

$S S \_$pipeline subsegment

\section{Table captions:}

Table 1: Coefficients and base cost data used in the application example calculations

Table 2: Design and real estate factors used in the application example calculations

Table 3: Final design statistics for cases A and B (Markov chain length 20)

\section{Figure captions:}

Figure 1: Pipeline system optimisation framework

Figure 2: Example of a current pipeline design

Figure 3: Illustration of pipeline perturbations

Figure 4: Geographical data used in the application example

Figure 5: Convergence results (design case A)

Figure 6: Final designs for case A (Markov chain lengths 6,10, 20 and 30)

Figure 7: Final designs for case B (Markov chain length 20)

Figure 8: Final profiles for pipe wall thickness 


\section{Tables}

Table 1: Coefficients and base cost data used in the application example calculations

\begin{tabular}{|c|c|c|c|c|}
\hline Symbol & Description & Value & Units & Source \\
\hline$a_{D}$ & coefficient for pump driver cost & 1.00 & - & $*$ \\
\hline$a_{P}$ & coefficient for main pump cost & 0.67 & - & * \\
\hline$a_{M}$ & coefficient for pump maintenance cost & 0.08 & - & $*$ \\
\hline$a_{R}$ & coefficient for pump repair cost & 0.25 & - & $*$ \\
\hline$b_{M}$ & coefficient for pipe maintenance cost & 0.01875 & $\mathrm{USD} / \mathrm{m} / \mathrm{inch}$ & $*$ \\
\hline$c_{F}$ & natural gas unit price & 0.2306 & $\mathrm{USD} / \mathrm{m}^{3}$ & ** \\
\hline$c_{s}$ & steel unit price & 1.2 & $\mathrm{USD} / \mathrm{kg}$ & $*$ \\
\hline$C P_{B, D}$ & base pump driver cost & $1.3 \times 10^{6}$ & USD & $*$ \\
\hline$C P_{B, P}$ & base main pump cost & $1.0 \times 10^{6}$ & USD & $*$ \\
\hline$C S_{B, L}$ & base land cost coefficient & 100 & $\mathrm{USD} / \mathrm{m}$ & $*$ \\
\hline$d$ & internal pipe diameter & 19.25 & inches & \\
\hline$g$ & gravitational force & 9.81 & $\mathrm{~m} / \mathrm{s}^{2}$ & \\
\hline$H_{B}$ & base pump head & 650 & $\mathrm{~m}$ & * \\
\hline$H V_{F}$ & natural gas heating value & $38.3 \times 10^{6}$ & $\mathrm{~J} / \mathrm{m}^{3}$ & $* *$ \\
\hline$N S_{, f}$ & pipe failure rate & $10^{-5}$ & $\mathrm{~m}^{-1} \cdot \mathrm{yr}^{-1}$ & $*$ \\
\hline$Q$ & oil volumetric flow rate & 160 & kBPD & \\
\hline$r$ & roughness (commercial steel) & $1.8 \times 10^{-3}$ & inches & $* * *$ \\
\hline$s$ & specified minimum yield strength & $4.480 \times 10^{5}$ & $\mathrm{Nt} / \mathrm{m}$ & $*$ \\
\hline$T$ & design operation lifetime & 20 & years & \\
\hline$T P_{f}$ & MTBF for pumps & 3 & years & * \\
\hline $\boldsymbol{\delta}_{i}\left(\boldsymbol{p}_{i}^{*}\right)$ & hazard distance & 20 & $\mathrm{~m}$ & $* * * *$ \\
\hline$\eta$ & pump energy efficiency & 0.80 & - & $*$ \\
\hline$\rho$ & oil density & 830 & $\mathrm{~kg} / \mathrm{m}^{3}$ & \\
\hline$\rho_{S}$ & steel density & 7850 & $\mathrm{~kg} / \mathrm{m}^{3}$ & $*$ \\
\hline$\tau$ & [unit conversion] & $3600 \times 24 \times 365$ & $\mathrm{~s} / \mathrm{yr}$ & \\
\hline
\end{tabular}

* derived from available past data

** Annual Energy Review (2009)

**** CRANE (2009)

***** Jo and Crowl (2008) 
Table 2: Design and real estate factors used in the application example calculations

\begin{tabular}{clcc}
\hline Factor & Brief description of location class & Value & Source \\
\hline $\boldsymbol{F}_{\boldsymbol{i}, \boldsymbol{I}}$ & class location 1, division 1 & 0.80 & $*$ \\
& class location 1, division 2 & 0.72 \\
& class location 2 & 0.60 \\
& class location 3 & 0.50 \\
& class location 4 & 0.40 \\
\hline $\boldsymbol{F}_{\boldsymbol{i}, \boldsymbol{C}}$ & cross country pipeline, including slope factor & $1+\Delta z_{A B} / L_{i} \cdot 0.15$ \\
& restricted working pipeline (e.g. forest) & 3.00 \\
& street works (inside sparsely populated areas) & 3.00 \\
& street works (inside densely populated areas) & 4.00 \\
& crossings (rivers, railways, national roads) & 5.00 \\
\hline \multirow{2}{*}{$\boldsymbol{F}_{\boldsymbol{i} \boldsymbol{L}}$} & class location 1 \& rural & - \\
& class location 2 & 1.0 \\
& class location 3 & 2.0 \\
& class location 4 & 7.2 \\
\hline
\end{tabular}

\footnotetext{
*ASME (2010)

*** derived from available past data
} 
Table 3: Final design statistics for cases A and B (Markov chain length 20)

\begin{tabular}{lrrrrrrrr}
\hline & \multicolumn{3}{c}{ Design case A } & \multicolumn{5}{c}{ Design case B } \\
\hline & Median & \multicolumn{1}{c}{$Q_{1}$} & \multicolumn{1}{c}{$Q_{3}$} & $Q V$ & Median & $Q_{1}$ & \multicolumn{1}{c}{$Q_{3}$} & $Q V$ \\
\hline investment and real estate (k\$) & 686 & 680 & 695 & $1.11 \%$ & 967 & 959 & 981 & $1.12 \%$ \\
construction cost (k\$) & 35,805 & 35,780 & 35,843 & $0.0875 \%$ & 38,383 & 38,342 & 38,433 & $0.118 \%$ \\
pipeline maintenance cost (k\$) & 308 & 308 & 308 & $0.0866 \%$ & 330 & 330 & 331 & $0.117 \%$ \\
pump investment (k\$) & 1,135 & 1,126 & 1,140 & $0.629 \%$ & 1,261 & 1,256 & 1,265 & $0.341 \%$ \\
operational cost (fuel) (k\$) & 3,207 & 3,176 & 3,224 & $0.754 \%$ & 3,637 & 3,621 & 3,650 & $0.407 \%$ \\
pump maintenance cost (k\$) & 3,708 & 3,678 & 3,724 & $0.629 \%$ & 4,118 & 4,103 & 4,131 & $0.341 \%$ \\
\hline total cost (k\$) & 44,855 & 44,786 & 44,924 & $0.153 \%$ & 48,721 & 48,639 & 48,821 & $0.187 \%$ \\
\hline total pipeline length (m) & 41,474 & 41,447 & 41,521 & $0.0886 \%$ & 44,379 & 44,328 & 44,438 & $0.124 \%$ \\
number of simulations & 19,069 & 17,662 & 20,657 & $7.82 \%$ & 18,613 & 18,609 & 18,661 & $0.140 \%$ \\
\hline
\end{tabular}


Figure 1: Pipeline system optimisation framework

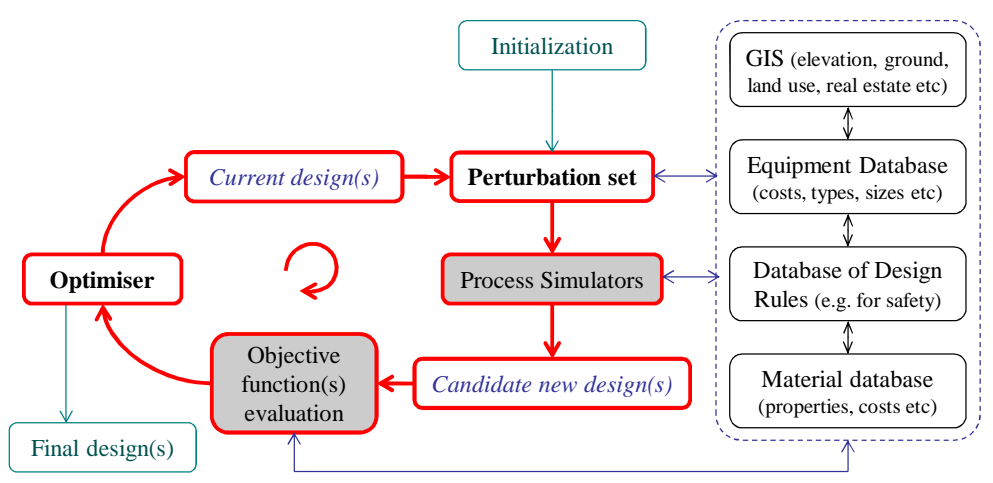

Figure 2: Example of a current pipeline design

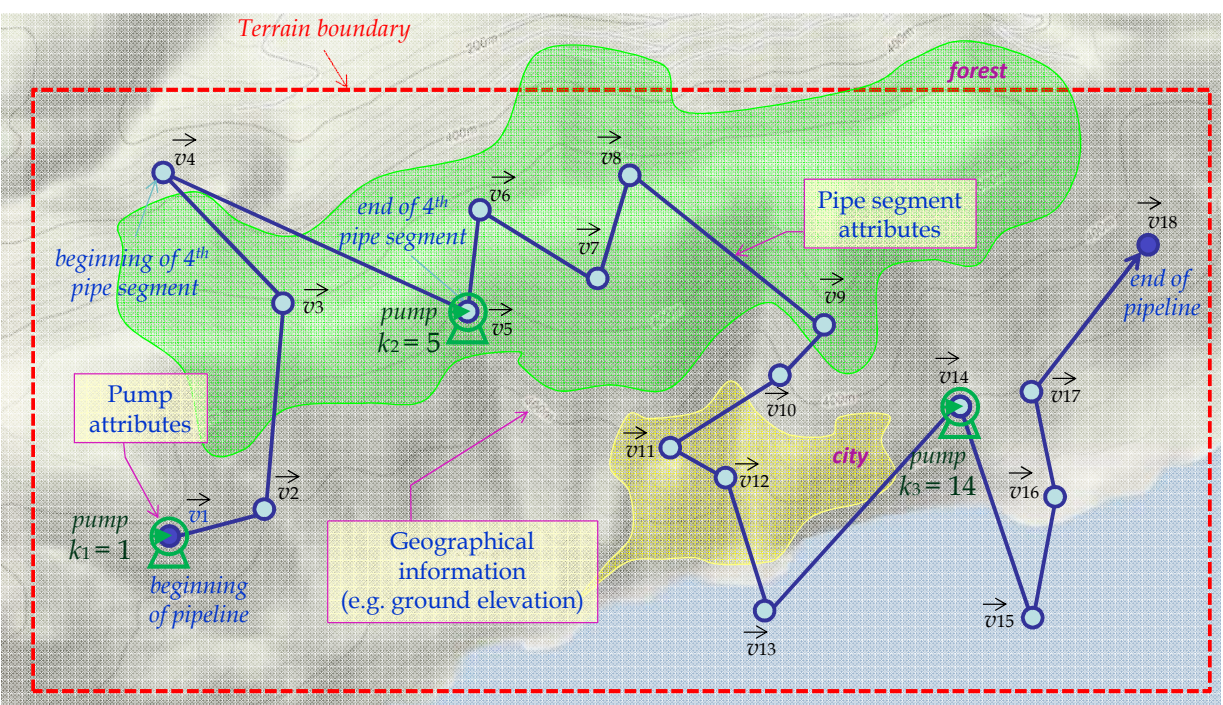


Figure 3: Illustration of pipeline perturbations

(a) Remove an existing point (plain point)

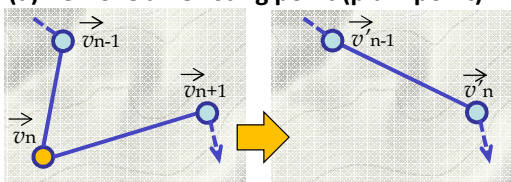

(c) Relocate an existing point (plain point)

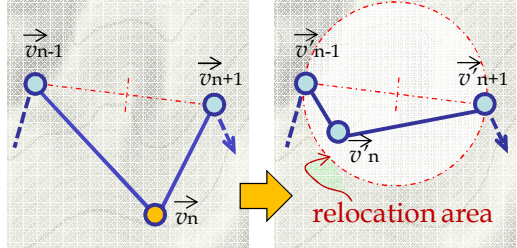

(e) Add a new point

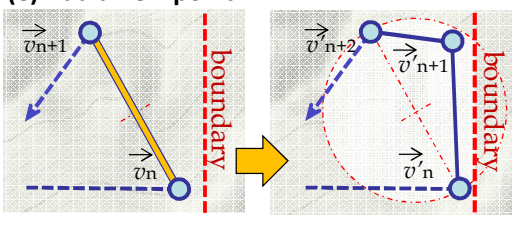

(b) Remove an existing point (with $P C D$ )

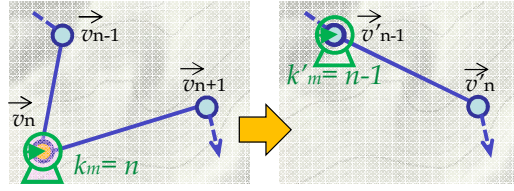

(d) Relocate an existing point (with PCD)

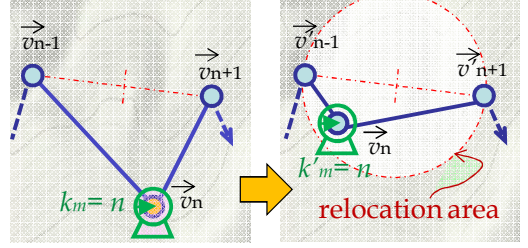

(f) Add / remove PCD's

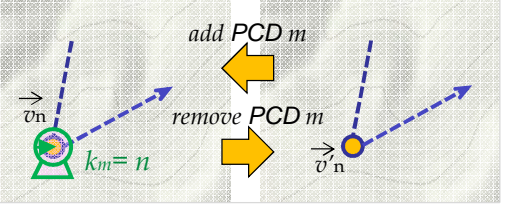

Figure 4: Geographical data used in the application example

(a) Ground elevation data used in design cases $A$ and $B$

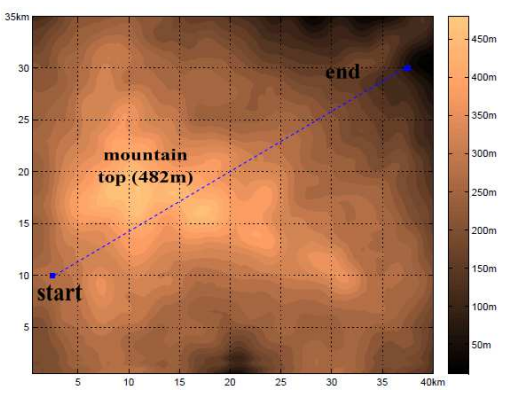

(b) Location data for design factor calculations used in design case $B$

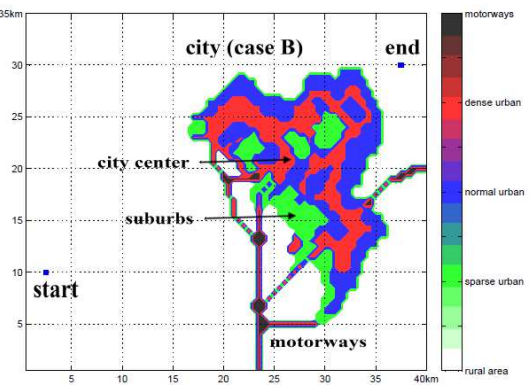


Figure 5: Convergence results (design case A)

(a) Simulations vs. chain length

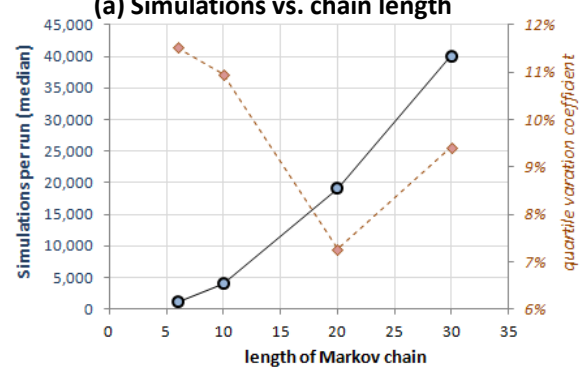

(b) Pipeline length vs. chain length

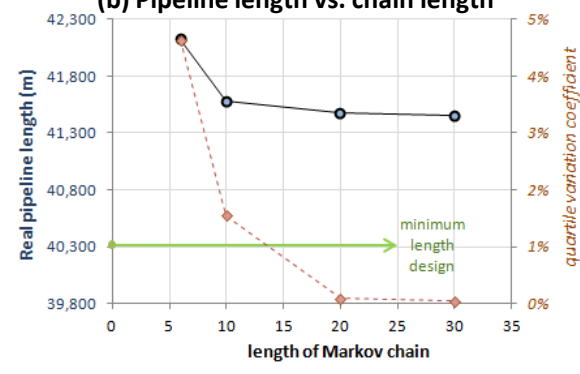

(c) Total cost vs. chain length

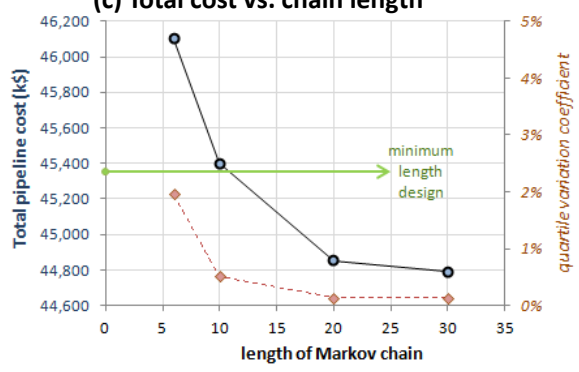

Figure 6: Final designs for case A (Markov chain lengths 6,10, 20 and 30)
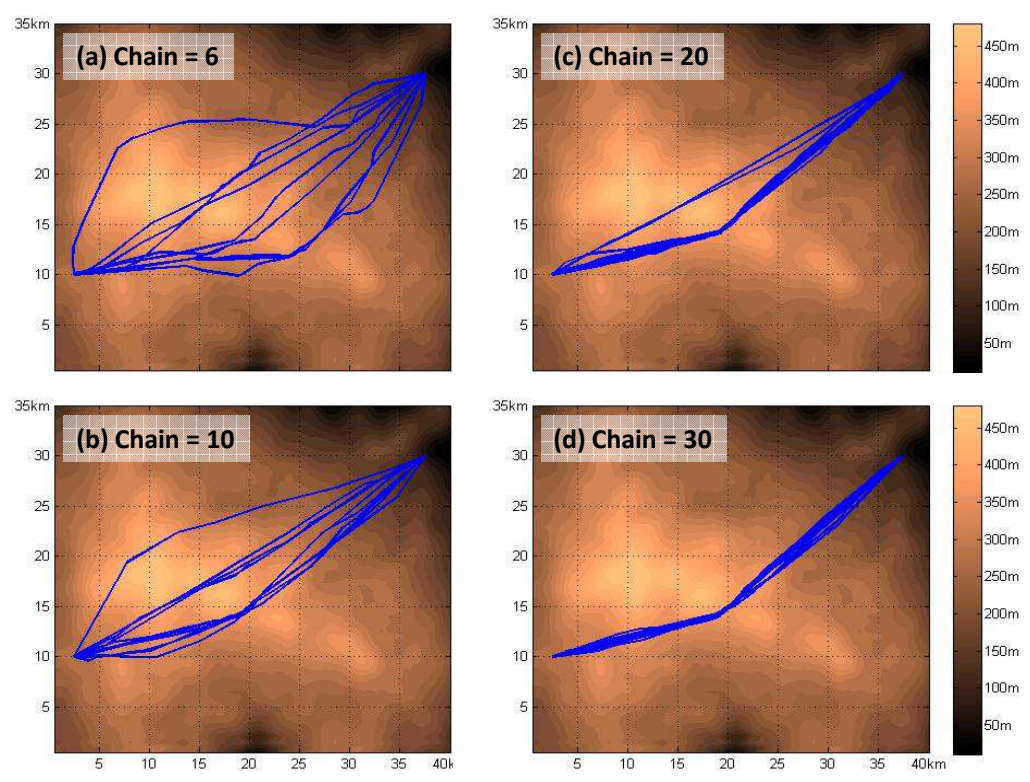
Figure 7: Final designs for case B (Markov chain length 20)

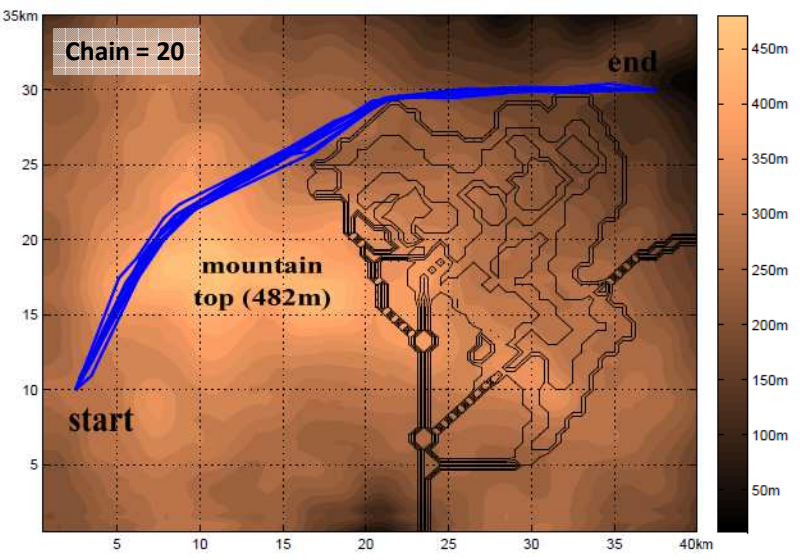

Figure 8: Final profiles for pipe wall thickness

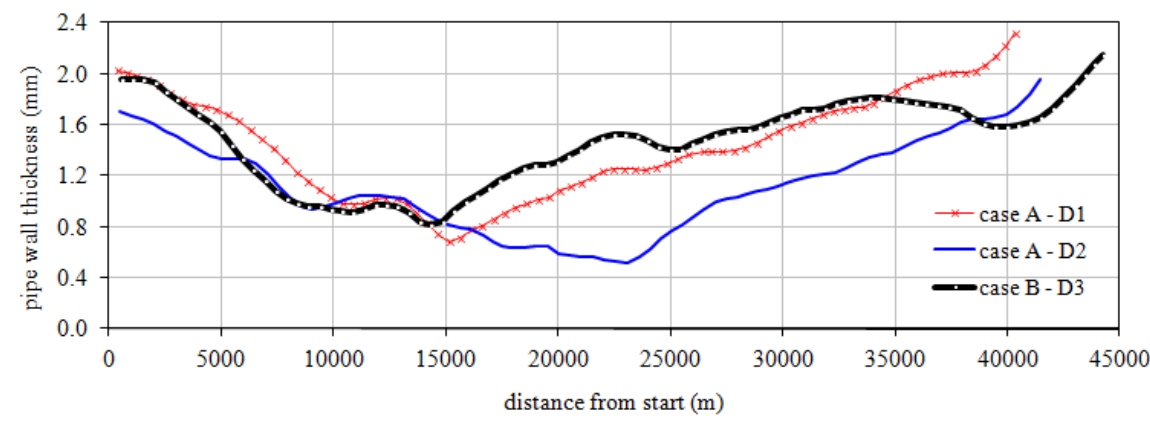

